\title{
Performance Evaluation Analysis of Wavelength/Time 2-D Modified Double Weight Code in OCDMA
}

\author{
Israa Sh. Ahmed ${ }^{1}$, S.A. Aljunid ${ }^{2}$, C. B. M. Rashidi ${ }^{3}$, Layth A.Khalil ${ }^{4}$
}

\begin{abstract}
The work in this paper a simulation of two-dimension (2-D) modified double weight (MDW) optical code division multiple access (OCDMA) wavelength-time and 2-D difference perfect code PDC, focusing on a comparison between the two codes with $k_{1}=4$ (the code weight of spectra code sequence), $k_{2}=2$ (the code weight of time-chip) for 2-D MDW code and $k_{1}=3, k_{2}=2$ for 2-D PDC with four users. The simulation is done with Optisystem program V11, can notice that 2-D MDW code indicates substantial performance improvements in bit error rate (BER), data rate and distance and achieves good performance; below $10^{-9}$ BER error floor for lowest effective transmitted power $\left(\mathrm{P}_{s r}\right)$ reached $-20 \mathrm{~dB}$ and successfully suppresses PIIIN and mitigating MAI compared with PDC.
\end{abstract}

Keywords-One Dimension Modified Double weight (1DMDW). Two Dimension Modified Double Weight (2D-MDW), Optical Code Division Multiple Access (OCDMA), Phase Induced Intensity Noise (PIIN), Multiple Access Interference MAI.

\section{INTRODUCTION}

2 -D OCDMA emerging research attracts huge research interest, it offers a large optical fiber bandwidth and flexibility of high speed access, dynamic bandwidth assignment and high security [1]. The challenges that can see in OCDMA system are improved cardinality, suppress phase induced intensity noise PIIN, and mitigate multiple access interference MAI [2]. However a proper design of 2-D OCDMA could result in auto-correlation with minimum or zero side lobe and cross-correlation small than 1-D OCMA [3].

The high weight and short length result in a high signal to noise ratio (SNR) and low BER therefore various 2-D OCDMA techniques have been developed in designing the code sequences with emphasizing on specific code properties, headed for suppressing interferences and enhancing BER [4]. 2-D wavelength-time OCDMA system is anticipated to be an alternative to the next generation OCDMA system [5]. There are some factors contributing towards improvement OCDMA

\footnotetext{
${ }^{1,2,3,4}$ School of Computer \& Communication Engineering, Universiti Malaysia Perlis (UniMAP) Tingkat 1, Kampus Tetap Pauh Putra, 02600 Arau, Perlis

${ }^{1,2,3}$ Center of Excellent Advanced Communication Engineering, School of Computer \& Communication Engineering, Universiti Malaysia Perlis (UniMAP), 01000 kangar, Perlis.
}

code sequences system performance, cross-correlation and auto-correlation properties, detection technique and BER and important to mention that the increment in code weight will improve the correlation properties, SNR and BER [5].

Many new 2-D OCDMA codes have been developed such as 2-D Hybrid FCC MDW code, which has a good correlation properties, and have ability to suppress MAI [6], Choe code, which possessed larger capacity and good spectral efficiency [7], 2-D Perfect Difference (PDC), eliminate the MAI and improve the system performance by suppressing the PIIN [8]. 2-D MDW code which have fixed in-phase spectral crosscorrelation equal to ' 1 ' signature sequences to reduce the effect of PIIN and removing MAI and offers an enhanced performance for the family of MDW codes[9]. 2-D MDW code is envisioned as a possible solution to overcome the limitation of 1-D wavelength -time OCDMA codes that improved a high numbers of users a result BER improvement [10].

The structure of this paper is starting with 2-D MDW code construction in section 2, section 3 will be the crosscorrelation of the code. The system setup in section and simulation result in section 4.finally the conclusion in section 5.

\section{ESSENTIAL OF 2-D MDW CODE ALGORITHM}

All 2-D MDW codes are developed from 1-D MDW code [6] , where $M$ as number of wavelengths , $N$ as temporal code length, $W$ as a weight , $\lambda^{\lceil a\rceil}$ and $\lambda^{\lceil c\rceil}$ are auto- and cross-correlation values are denoted by $\left(M \times N, W, \lambda^{\lceil a\rceil}\right.$, $\lambda^{\lceil c\rceil}$ ) the jth user's 2-D codes $C_{M, N}^{j}$ in Eq.(1), is a matrix $M$ of row vectors $d_{K, N}^{j}$ related to the temporal spreading $d_{1, N}^{j}=\left[C_{K, 1}^{j}, C_{K, 2}^{j}, \ldots \ldots \ldots ., C_{K, N-1}^{j}, C_{K, N}^{j}\right]$ where $C_{K, i}^{j} \epsilon$ $\{0,1\}$ and $K$ is the emitted wavelength of $K \epsilon$ $\{1, \ldots \ldots, M\}$. 


$$
C_{M, N}^{j}=\left(\begin{array}{l}
\mathrm{d}_{1, N}^{j} \\
\mathrm{~d}_{2, N}^{j} \\
- \\
- \\
- \\
\mathrm{d}_{M-1, N}^{j} \\
\mathrm{~d}_{M, N}^{j}
\end{array}\right)
$$

The signals $r_{K, N}(t)$ are sum of temporal spreading data of $\mathrm{F}^{u}$ users carried on the wavelength $\lambda^{k}$ and expressed as $r_{K, N}(t)=\sum_{i=1}^{F^{u}} b_{i}^{j}(t) d_{K, N}^{j}$, where (t) is ith data bit jth user and $F u$ is number of users. The $M$ signals $r_{K, N}^{j}(t)$ are multiplexed and total signal $R_{M, N}(t)$ transmitted on optical fiber is expressed as a matrix of $(M \times N)$ :

$$
R_{M, N}(t)=\left(\begin{array}{c}
r_{1, N}(t) \\
r_{2, N}(t) \\
\cdot \\
- \\
r_{M-1, N}(t) \\
r_{M, N}(t)
\end{array}\right)
$$

2-D MDW OCDMA network consists of $M, N$ pairs of transmitters and receivers. $A^{g, h}$ represents the code where $\mathrm{g}$ $\in(1,2,3, \ldots \ldots, M-1)$ and $\mathrm{h} \in(1,2,3, \ldots \ldots, N-1)$ . $X^{g}$ and $Y^{k}$ are spectral and spatial encoding. Table 1 shows some 2-D MDW code projection.

TABLE I

2-D MDW CODES FOR $k_{1}=4$ AND $k_{2}=2$

\begin{tabular}{llll}
\hline$X_{g, h}$ & {$[000011011]$} & {$[011000110]$} & {$[110110000]$} \\
0 & 000000000 & 000000000 & 000000000 \\
1 & 000011011 & 011000110 & 110110000 \\
1 & 000011011 & 011000110 & 110110000 \\
1 & 000011011 & 011000110 & 110110000 \\
1 & 000011011 & 011000110 & 110110000 \\
0 & 000000000 & 000000000 & 000000000 \\
\hline
\end{tabular}

\section{THE 2-D MDW CROSS-CORRELATION}

$X=\left[\mathrm{x}^{0}, \mathrm{x}^{1}, \mathrm{x}^{2} \ldots ., \mathrm{x}^{M-1}\right], Y=\left[\mathrm{y}^{0}, \mathrm{y}^{1}, \mathrm{y}^{2}, \ldots . . \mathrm{y}^{N-1}\right]$ are code sequences of 1-D MDW code. The 2-D MDW crosscorrelation can be derived through four characteristic matrices:

$A^{(d)}$, where $\mathrm{d} \in(0,1,2,3)$ are defined as $A^{(0)}=Y^{T} X$, $A^{(1)}=Y^{T} \bar{X}$ and $A^{(2)}=\bar{Y} X$ and $A^{(3)}=\bar{Y}^{T} \bar{X}$. The cross-correlation of 2-D MDW code $A^{(d)}$ and $A_{g, h}$ can be expressed as:

$R^{(d)}(\mathrm{g}, \mathrm{h})=\sum_{i=0}^{M-1} \sum_{j=0}^{N-1} a_{i j}^{(d)} a_{(i+g)(j+h)}$, where $a_{i j}^{(d)}$ is $(\mathrm{i}, \mathrm{j})$ th of $A^{(d)}$ and $a_{(i+g)(j+h)}$ is $(\mathrm{i}, \mathrm{j})$ th of $A_{g, h}$ :

$\mathrm{g} \in(1,2,3, . ., M-1)$ and $\mathrm{h} \in(1,2,3, \ldots \ldots, N-1)$.

Table 2 [6], shows the cross-correlation of 2-D MDW code generated from $R^{(d)}(\mathrm{g}, \mathrm{h})$, where $R^{(3)}$ (g,h) has nonzero value when $\mathrm{g} \neq 0 \cap \mathrm{h} \neq 0$.

TABLE II

2-D MDW CODE CROSS-CORRELATION

\begin{tabular}{ccccc}
\hline$X_{g . h}$ & $R_{(g, h)}^{(0)}$ & $R_{(g, h)}^{(1)}$ & $R_{(g, h)}^{(2)}$ & $R_{(g, h)}^{(3)}$ \\
\hline $\mathrm{g}=0, \mathrm{~h}=0$ & $k_{1} k_{2}$ & 0 & 0 & 0 \\
$\mathrm{~g}=0, \mathrm{~h} \neq 0$ & $k_{1}$ & 0 & 0 \\
$\mathrm{~g} \neq 0, \mathrm{~h}=0$ & $k_{1}$ & 0 & $k_{2}\left(k_{1}-1\right)$ & 0 \\
$\mathrm{~g} \neq 0, \mathrm{~h} \neq 0$ & $k_{2}$ & 1 & $k_{1}-1$ & $k_{1}-1$ \\
& 1 & & & \\
\hline
\end{tabular}

When $\mathrm{g} \neq 0 \cap \mathrm{h} \neq 0 ; \quad R^{(1)}(\mathrm{g}, \mathrm{h}), \quad R^{(2)}(\mathrm{g}, \mathrm{h})$ and $R^{(3)}(\mathrm{g}, \mathrm{h})$ indicates the specific relationships . Cross-correlation function can be written by using $R^{(3)}(\mathrm{g}, \mathrm{h})$ to eliminate influence due to $A_{g, h}$ from $R^{(0)}(\mathrm{g}, \mathrm{h}), R^{(1)}(\mathrm{g}, \mathrm{h})$ and $R^{(2)}$ (g,h) when $\mathrm{g} \neq 0 \cap \mathrm{h} \neq 0$. New derived 2-D MDW crosscorrelation function is expressed as:

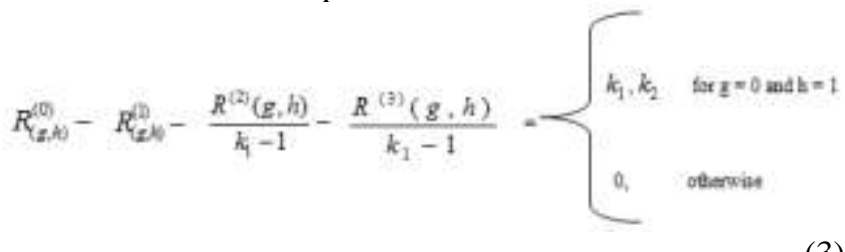

\section{2- D MDW COdE Simulation SETUP AND RESUlts}

The result of simulation for the 2-D MDW OCDMA system compared with 2-D PDC OCDMA of four users and eye pattern are shown in the figures below. The wavelength chip is set to an increment of $0.8 \mathrm{~nm}$ spectral width, with varied data rate for as distance varied using ITU-TG.652 standard single mode optical fiber and the attenuation $\alpha$ (i.e., $0.2 \mathrm{~dB} / \mathrm{km}$ ). The performance is measured based on the BERs and eye pattern analysis. As we varied the system, data rate of $622 \mathrm{Mbps}$ and $1 \mathrm{Gbps}$ with fiber length from $5 \mathrm{~km}$ to $30 \mathrm{~km}$ for four users. 
Figure 1 can shows the eye diagram for the 2-D MDW code with data rate of $622 \mathrm{Mbps}$ detected by the ANDsubtraction technique with $k_{1}=4, k_{2}=2$ for 2-D MDW code, where the BER value is $5 \times 10^{\wedge}-52$ for fibre length equal 10 $\mathrm{km}$. The high performances of the system are characterized by referring to the bit-error rate (BER) based on the properties of 2-D MDW code in term of length of the code and the cross-correlation.

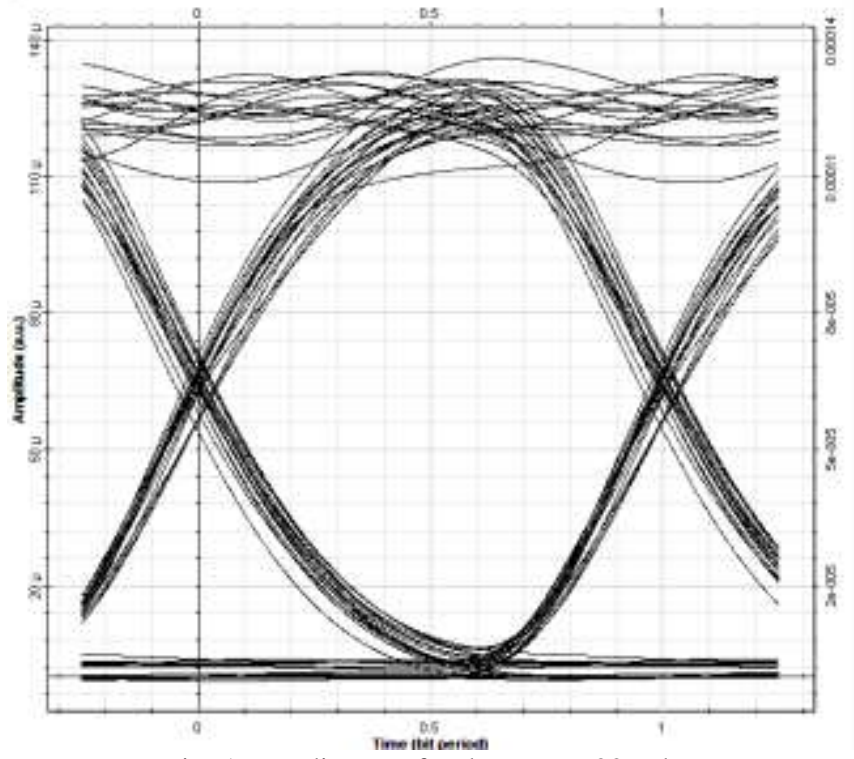

Fig. 1: eye diagram for data rate $622 \mathrm{Mbps}$

Fig. 2 shows the eye diagram for the 2-D MDW code with data rate of $1 \mathrm{Gbps}$ where the $\mathrm{BER}=7 \times 10^{\wedge}-17$ for the same transmission distance and using of AND-Subtraction detection technique. As the bit rate increases the vertical distance between the top of the eye opening and maximum signal level gives the degree of distortion. The more the eye closes, the more difficult it is to distinguish between $1 \mathrm{~s}$ and $0 \mathrm{~s}$ in the signal.

In figure 3 the simulation results depend on the relation between the data bit rate and the transmission distance at data bit rate of $622 \mathrm{Mbps}$ the 2-D MDW OCDMA system can reach $20 \mathrm{~km}$ below $10^{-9}$ BER. For 2-D PDC OCDMA can achieve BER equal to $10^{-9}$ with fiber length equal $16 \mathrm{~km}$, when increase transmission distance results in higher utilization of receive power and interference in the system and subsequently deteriorate the system performance. However, as the bit rate increases to data bit rate of $1 \mathrm{Gbps}$ for the 2-D MDW OCDMA network still below $10^{-9}$ for transmission distance equal $12 \mathrm{~km}$ and for 2-D PDC code the transmission distance equal $9 \mathrm{~km}$. Moreover when transmission distance $7 \mathrm{~km}, \mathrm{BER}=10^{-9}$ exactly of data bit rate $1.5 \mathrm{Gbps}$ for 2-D MDW code, for 2-D PDC OCDMA network BER can be $10^{-9}$ for bit rate equal to 1.5 Gbps at the transmission distance $6 \mathrm{~km} .2-\mathrm{D}$ MDW. Finally this result clearly shows that the 2-D MDW code is better than 2-D PDC for high bit rate under long transmission distance.

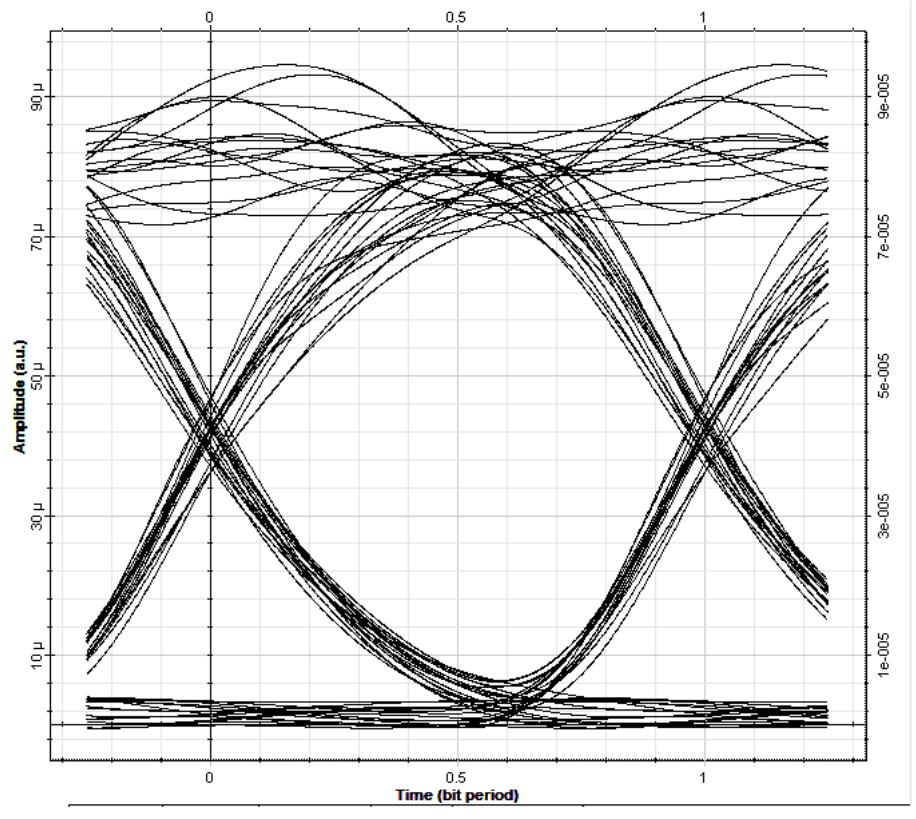

Fig 2: eye diagrm for data rate $1 \mathrm{Gbps}$

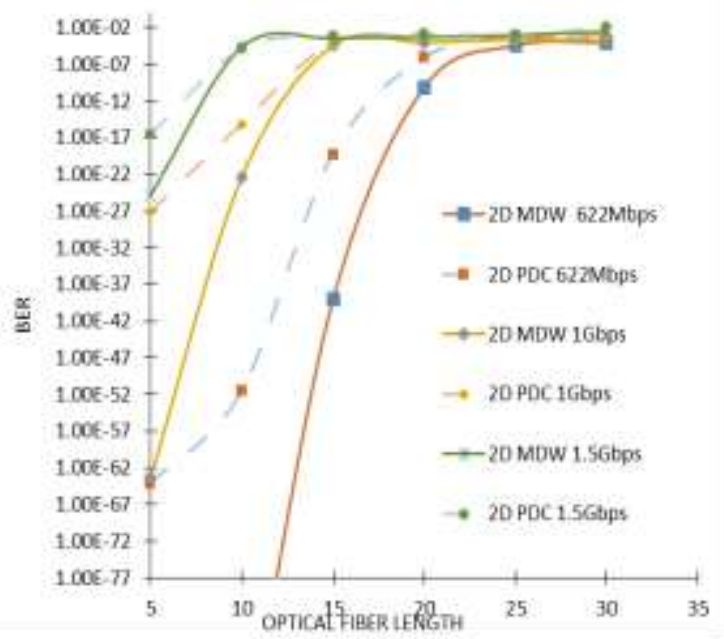

Fig. 3: Performance simulation of BER versus fiber length of 2-D MDW and 2-D PDC

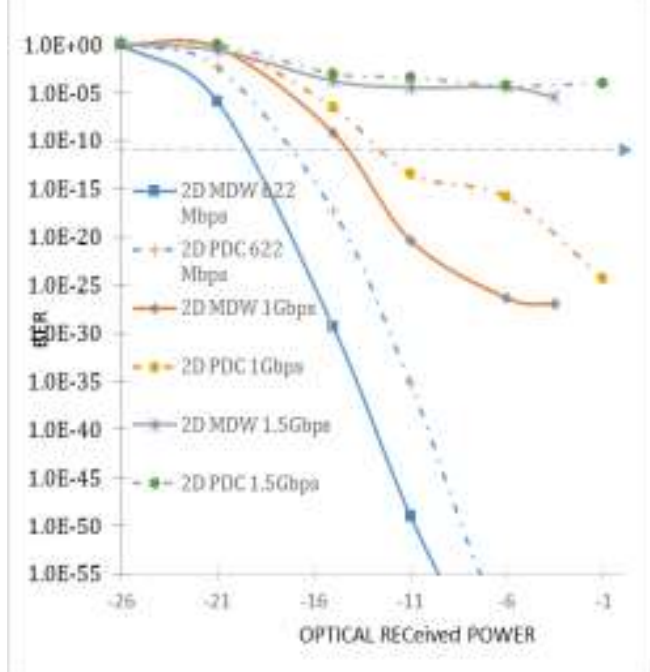

Fig. 4: BER versus $\mathrm{P}_{s r}$ with four numbers of users with different bit rates 
As shown in fig. 4 the BER versus $\mathrm{P}^{s r}$ with four numbers of users of 2-D MDW OCDMA and 2-D PDC OCDMA for different data bit rates $622 \mathrm{Mbps}, 1 \mathrm{Gbps}$ and $1.5 \mathrm{Gbps}$, with transmission distance $10 \mathrm{Km}$ distance. For 2D MDW meet the optical transmission requirement with lowest effective transmitted source power $\mathrm{P}^{s r}$ at $-20 \mathrm{dBm}$ compare to 2-D PDC OCDMA at $622 \mathrm{Mbps}$ the effective received power equal $-17 \mathrm{dBm}$, but when increase the bit rate to $1 \mathrm{Gbps} \mathrm{P}^{s r}$ equal $-14 \mathrm{dBm}$ for 2-D MDW OCDMA but for 2-D PDC $\mathrm{P}^{s r}$ equal $-11 \mathrm{dBm}$ that mean for this bit rate 2-D MDW OCDMA receive a clear signal in low effective power. However, for $1.5 \mathrm{Gbps}$ and sure for highest bit rates the both of codes $\mathrm{P}^{s r}$ is above $10^{-9}$ BER error. Depend on this results the 2-D MDW has advantage over the 2-D PDC due to the ability to extremely suppress PIIN. This is achieved through good property of minimum cross-correlation.

\section{V.CONCLUSION}

Resolution can be made from the simulation for 2-D MDW improved the good performance at high bit rates with different optical fiber length and receive power that can be $20 \mathrm{dBm}$ comparing with 2-D PDC. 2-D PDC code code has a good performance simulation analysis and in-phase crosscorrelation code sequences are exactly one, but has disadvantage it's required lengthy code as the number of the user increases. 2-D MDW result's scheme has better tolerance to PIIN, the code properties restrain the system degradation.

\section{REFERENCES}

[1] Aljunid, S. A., Hasson, F. N., Samad, M. D. A., Abdullah, M. K., Othman, M., \& Shaari, S. (2006, October). Performance of OCDMA systems using AND subtraction technique. In 2006 IEEE International Conference on Semiconductor Electronics. http://dx.doi.org/10.1109/SMELEC.2006.381104

[2] Zahid, A. Z. G., Mandeep, J. S., Menon, P. S., Bakarman, H., Hasoon, F. N., Bakar, A. A. A., \& Ali, M. A. M. (2012, October). Performance analysis of multi-weight 2D-OCDMA TEDW. In Photonics (ICP), 2012 IEEE 3rd International Conference on (pp. 206-209). IEEE.

[3] Rashidi, C. B. M., Aljunid, S. A., Ghani, F., Fadhil, H. A., \& Anuar, M. S. (2012). Cardinality Enhancement Using Flexible Cross Correlation (FCC) Code for Spectral Amplitude Coding Optical Code Division Multiple Access Systems. Journal of Applied Sciences Research, 8(12), 5616$5626 \mathrm{R}$

[4] Morelle, M., Julien-Vergonjanne, A., Cances, J. P., \& Dumas, J. M. (2008, May). 2D Optical CDMA technique dedicated to Multimedia transmission. In Communications, 2008. ICC'08. IEEE International Conference on (pp. 5412-5416). IEEE.

[5] Arief, A. R., Aljunid, S. A., Anuar, M. S., Junita, M. N., \& Ahmad, R. B. (2013). Cardinality enhancement of spectral/spatial modified double weight code optical code division multi-access system by PIIN suppression. Optik-International Journal for Light and Electron Optics, 126(19), 37863793. http://dx.doi.org/10.1016/j.ijleo.2012.11.061

[6] Din Keraf, N., Aljunid, S. A., Arief, A. R., Anuar, M. S., Rashidi, C. B. M., Ehkan, P., \& Nurol, M. N. (2014, August). An optimal cardinality of wavelength/time incoherent OCDMA system using 2-D hybrid FCCMDW code. In Electronic Design (ICED), 2014 2nd International Conference on (pp. 356-361). IEEE.

[7] Zhang, Q., Xin, X., Wang, Y., Zhang, L., Yu, C., Meng, N., \& Wang, H. (2012, November). A novel 2D wavelength-time chaos code in optical CDMA system. In Photonics Asia (pp. 85561P-85561P). International Society for Optics and Photonics. http://dx.doi.org/10.1117/12.2001279
[8] Lin, Cheing-Hong, Jingshown $\mathrm{Wu}$, and Chun-Liang Yang. "Noncoherent spatial/spectral optical CDMA system with two-dimensional perfect difference codes." Journal of lightwave technology 23.12 (2005): 3966.

[9] C.-S. Weng and J. Wu, "Perfect difference codes for synchronous fiberoptic CDMA communication systems," J. Lightw. Technol., vol. 19, no. 2, pp. 186-196, Feb. 2001. http://dx.doi.org/10.1109/50.917875

[10] X. Zhou, H. H. M. Shalaby, C. Lu, and T. Cheng, "Code for spectralamplitude coding optical CDMA systems," Electron. Lett., vol. 36, no. 8, pp. 728-729, Apr. 13, 2000 http://dx.doi.org/10.1049/el:20000567

[11] Arief. A. R., Aljunid, S. A., Safar, A. M., Nordin, J. M., Ahmad, R. B., \& Ghani, F. "A New Family of Two-Dimensional Modified Doubled Weight Code Optical Code Division Multiple Access for Capacity Enhancement". (2012). 